\title{
TOWARDS AN IMPLEMENTATION OF BLOCKCHAIN-BASED TRANSCRIPTS WITH NOSQL DATABASES
}

\author{
Timothy Arndt \\ Department of Information Systems, Cleveland State University, Cleveland, OH, USA
}

\begin{abstract}
Blockchain has emerged as a transformative technology, from its beginning as the basis of cryptocurrencies to wider applications in areas such as property registration and insurance due to its characteristic as a distributed ledger which can remove the need for a trusted third party to facilitate transaction. This spread of the technology to new application areas has been driven by the development of smart contracts - blockchain-based protocols which can automatically enforce a contract. One area where the types of problems being considered for blockchain exists is higher education. Students in higher education are increasingly mobile, and in an ever more agile world, the friction and delays caused by multiple levels of administration in higher education can cause many anxieties and hardships for students. Distance learning as a primary platform for higher education promises to open up higher education to a wider range of learners than ever before. In this paper, we sketch initial implementations of a blockchain-based university transcript system in order to empower students and better fit today's ever more agile society.
\end{abstract}

\section{KEYWORDS}

Blockchain; NoSQL Databases; Higher Education

\section{INTRODUCTION}

Blockchain burst onto the scene with a paper published by the pseudonymous Satoshi Nakamoto in 2008 and was subsequently incorporated as part of the architecture of the cryptocurrency bitcoin in the following year. Blockchain, which is an open, distributed ledger that can efficiently record transactions between two parties in a verifiable and permanent way and which can also be programmed (via so-called smart contracts) to trigger transactions automatically (Iansiti and Lakhani, 2017). As a foundational technology (like TCP/IP), blockchain has been used or proposed for use in applications far beyond cryptocurrencies, including banking (Peters and Panayi, 2016), land registration - especially in developing countries (Underwood, 2016), insurance (Lamberti, F., et al., 2017), and online voting (Ayed, 2017). In this paper, we sketch our initial results on implementation of a system for another promising area for blockchain technology - higher education records (university transcripts).

\section{DISCUSSION}

In today's world, learners are more mobile than ever before. University students move from one university to another, both within one country as well as between countries. This may happen within a single degree program (student starts at a community college, moves to 4-year college $\mathrm{X}$ and then transfers to university $\mathrm{Y}$ where she completes her degree), or when completing one program and moving on to another (student completes his undergraduate degree at institute $\mathrm{X}$ in country $\mathrm{Y}$ and then starts a graduate program at institute $\mathrm{W}$ in country Z). Students today expect to be able to do this with a minimum of difficulty (for instance, credits for courses already taken should be transferred to the new institution so those courses don't have to be repeated). Blockchain technology can be used to ease these processes. 
More information on our approach can be found in a previous paper (Arndt, 2018). In the rest of this paper, we sketch new results - namely prototype implementations.

\section{IMPLEMENTATION}

In this section we will be sketch initial implementation of a prototype system for university transcripts which empower students using blockchain and NoSQL databases.

Ethereum is a promising platform for implementation of this system since it is an open-source, public, blockchain-based distributed computing platform and operating system featuring smart contract (scripting) functionality. In our system, the university transcript data can be stored in blocks and functionality on the transcripts implemented via smart contracts. We have explored this possibility and enhanced it by hooking to the Neo4j open-source NoSQL database. Neo4j is a native graph database since it implements the property graph model down to the storage level. A graph database fits well with the structure of the blockchain and can be used to interrogate and investigate parts of the blockchain using the cypher query language. The architecture of a prototype system using Ethereum and Neo4j is shown below.

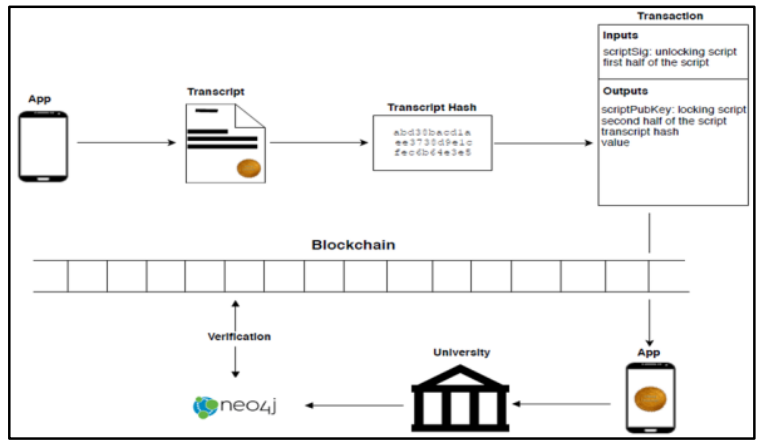

Figure 1. Neo4j/Ethereum-based prototype system

An example query is: MATCH (a:tx), (b:block) RETURN $a, b$. The result of the query is a graph, as shown below in figure 2 .

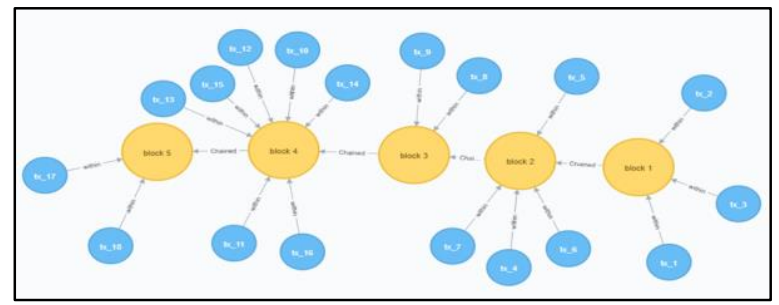

Figure 2. Query results

In the prototype, a small, relevant portion of the blockchain is imported into Neo4j as shown below in figure 3 .

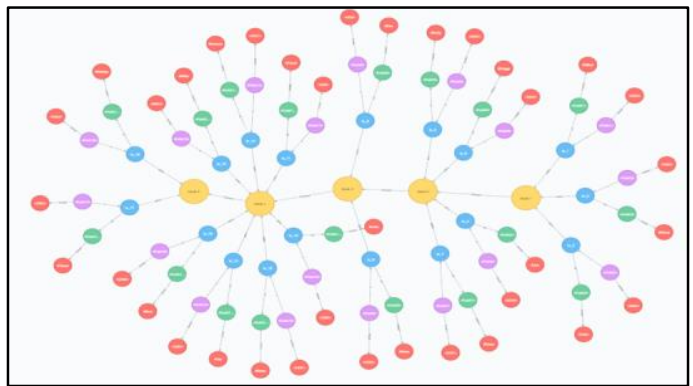

Figure 3. A portion of the blockchain imported into Neo4j database 
We can use Neo4j's cypher query language, e.g., to find nodes related to a student user Julie, with the following query, resulting in the results shown in figure 4. Query: MATCH $(a: t x),(b: b l o c k)<-$ $[$ :within]-(a:tx), (c:output)<-[:out]-(a:tx),

(d:input) $-[$ : in $]->(a:$ tx $),($ e:fromaddress $)<-[:$ locked $]-(d:$ input $), \quad$ (f:toaddress $)<-$ [:locked]-(c:output) WHERE b.blockhash = 'block 4' AND c.transcript = '\#Julie' RETURN $a, b, c, d, e, f$

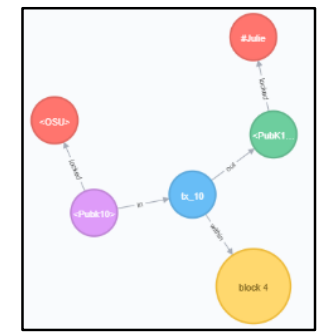

Figure 4. Results of query

Another approach we are exploring is the use of BigChainDB to securely store and retrieve student transcript records. BigchainDB is a hybrid model merging blockchain and Ddistributed DB systems. The architecture of the prototype system using this approach is in figure 5 below.

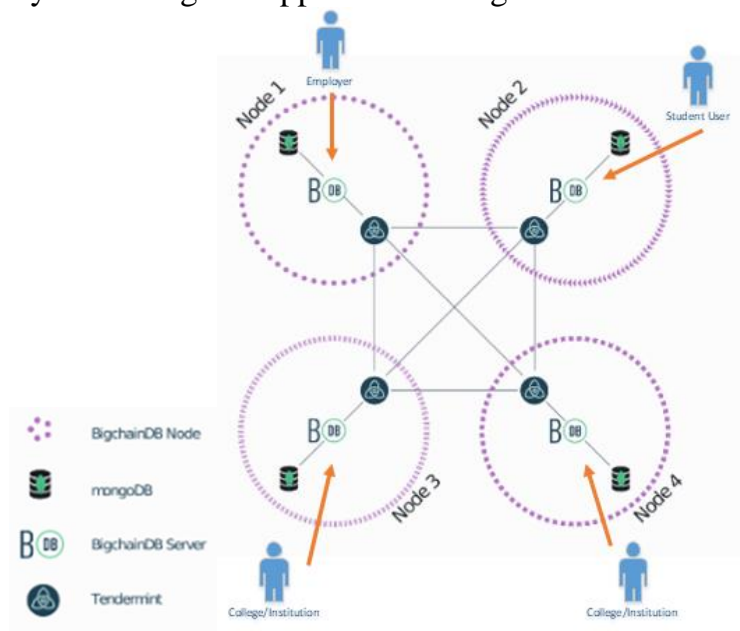

Figure 5. Architecture of the BigChainDB-based prototype system

A typical transcript process in this prototype system is shown in figure 6 below.

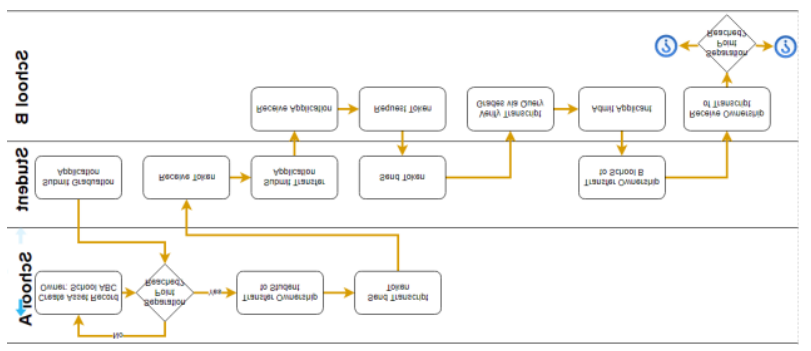

Figure 6. Transcript process in the prototype system

In this system, the transcript data is stored in BigChainDB's blockchain metadata. Transfer of transcript from a student to a university when the student enrolls at the university (so that the university may add data to the student's transcript) is shown below. The ownership of the transcript will be transferred back to the student when he separates from the university. 

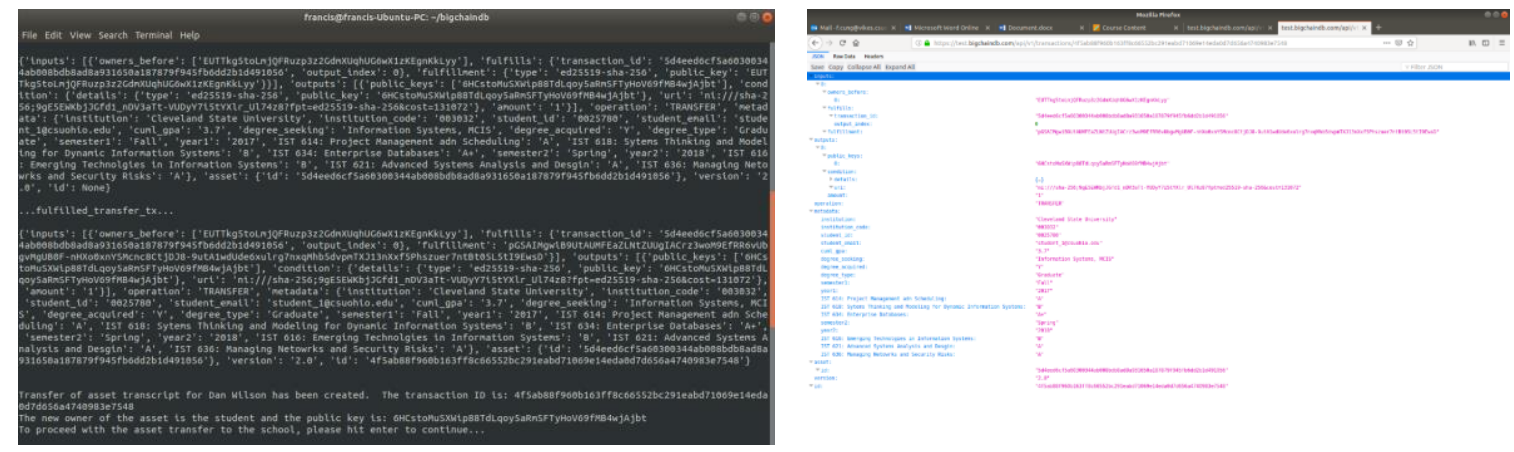

Figure 7. Transfer of transcript and the transfer transaction block created

Initial results of the implementation are encouraging. We are able to store transcript data in the blockchain and manipulate it using NoSQL database functionality. Update and transfer of the transcript data is supported. We will be adding functionality to the systems and scaling them up with larger amounts of data in the near future. Evaluation and development of the two prototype systems is ongoing.

\section{ACKNOWLEDGEMENT}

The help of students in the Master's of Information Systems course IST 634 Enterprise Databases is gratefully acknowledged.

\section{REFERENCES}

Arndt, T. 2018. Empowering University Students with Blockchain-Based Transcripts, in Proceedings of CELDA 2018, Budapest, Hungary, October 21-23, 2018.

Ayed, A. B., 2017. A conceptual secure Blockchain-based electronic voting system. International Journal of Network Security \& Its Applications, 93.

Iansiti, M. and Lakhani, K. R., 2017. The truth about blockchain. Harvard Business Review, 95(1), 118-127.

Lamberti, F., Gatteschi, V., Demartini, C., Pranteda, C., and Santamaria, V., 2017. Blockchain or not blockchain, that is the question of the insurance and other sectors. IT Professional.

Peters, G. W. and Panayi, E., 2016. Understanding modern banking ledgers through blockchain technologies: Future of transaction processing and smart contracts on the internet of money. In Banking Beyond Banks and Money (pp. 239-278). Springer, Cham.

Underwood, S., 2016. Blockchain beyond bitcoin. Communications of the ACM, 59(11), 15-17. 